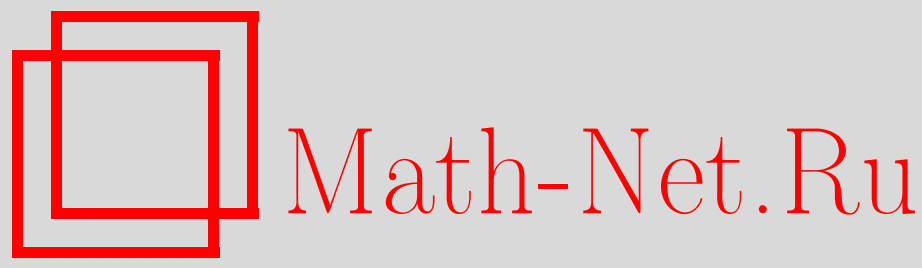

В. А. Градусов, С. Л. Яковлев, Об операторе Шредингера с суперпозицией короткодействующего и точечного потенциалов, ТMФ, 2015, том 183, номер 1, 90-104

DOI: https://doi.org/10.4213/tmf8798

Использование Общероссийского математического портала Math-Net.Ru подразумевает, что вы прочитали и согласны с пользовательским соглашением http://www.mathnet.ru/rus/agreement

Параметры загрузки:

IP : 54.198 .64 .247

26 апреля 2023 г., 12:01:43

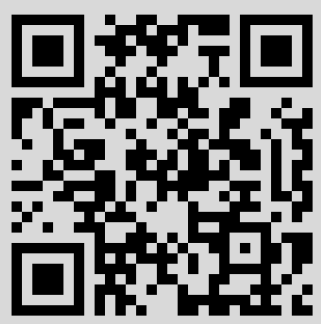




\section{ОБ ОПЕРАТОРЕ ШРЕДИНГЕРА С СУПЕРПОЗИЦИЕЙ КОРОТКОДЕЙСТВУЮЩЕГО И ТОЧЕЧНОГО ПОТЕНЦИАЛОВ}

Исследуется класс операторов Шредингера, для которых потенциальный член является суммой короткодействующего $V(\boldsymbol{r})$ и точечного потенциалов. Внимание уделено случаю, когда короткодействующий потенциал имеет особенность на носителе $r=0$ точечного взаимодействия. Точечное взаимодействие построено с помощью асимптотики при $\boldsymbol{r} \rightarrow 0$ функции Грина оператора Шредингера $-\Delta+V(\boldsymbol{r})$ с короткодействующим потенциалом $V$. Рассмотрены потенциалы, имеющие в начале координат особенность вида $r^{-\rho}$ с $\rho>0$. Исследование производится при помощи интегрального уравнения Липпмана-Швингера. Показано, что если особенность потенциала слабее, чем кулоновская, то асимптотика функции Грина имеет стандартное сингулярное поведение. В случае особенности потенциала вида $r^{-\rho}$ с $1 \leqslant \rho<3 / 2$ в асимптотике функции Грина возникает дополнительная сингулярность. В случае $\rho=1$ дополнительная логарифмическая сингулярность имеет ту же форму, что и в случае кулоновского потенциала. В случае $1<\rho<3 / 2$ дополнительная сингулярность имеет вид полярной особенности $r^{-\rho+1}$.

Ключевые слова: оператор Шредингера, точечное взаимодействие, псевдопотенциал, асимптотика функции Грина.

DOI: $10.4213 / \operatorname{tmf} 8798$

\section{1. ВВЕДЕНИЕ И ПОСТАНОВКА ЗАДАЧИ}

Точечным потенциалом называют взаимодействие, сосредоточенное в точке. В физической литературе такие потенциалы обычно называются потенциалами нулевого радиуса и широко используются для построения моделей межчастичных взаимодействий в квантовой механике [1]. Математически строгое определение точечного взаимодействия впервые было дано в работе Березина и Фаддеева [2]. Метод этой работы основан на теории самосопряженных расширений симметричных операторов. Дальнейшее развитие метода связано в основном с работами авторов монографии [3]. Интерес исследований затем сместился к более сложным, чем точечные,

${ }^{*}$ Санкт-Петербургский государственный университет, Санкт-Петербург, Россия. E-mail: v.gradusov@spbu.ru, s.yakovlev@spbu.ru 
контактным взаимодействиям, среди которых отметим точечные взаимодействия с внутренней структурой [4], [5], контактные взаимодействия, сосредоточенные на поверхностях, кривых и графах [6], [7]. Точечные потенциалы используются для описания взаимодействия частиц в гамильтонианах на решетке [8].

В настоящей работе мы рассматриваем случай одноцентрового точечного потенциала, который добавляется в гамильтониан системы двух частиц, взаимодействующих в трехмерном пространстве. Этот невозмущенный гамильтониан имеет вид

$$
H=-\Delta+V
$$

суммы оператора кинетической энергии $-\Delta$, где $\Delta-$ лапласиан, и локального потенциала $V$. Задача состоит в определении возмущенного точечным взаимодействием гамильтониана $\widetilde{H}$ формального вида $-\Delta+V+« \lambda \delta »$, где последнее слагаемое символически обозначает точечный потенциал с константой связи $\lambda$. Интерес представляет ситуация, в которой потенциал $V$ имеет сингулярность в точке сосредоточения точечного потенциала (без ограничения общности везде далее будем считать, что этой точкой является $r=0)$. Иначе потенциалы могут рассматриваться независимо, и точечный потенциал остается таким же, как в случае полного отсутствия короткодействующего взаимодействия [1].

Задача построения оператора $\widetilde{H}$ может быть решена в рамках метода самосопряженных расширений. Упомянем здесь несколько работ, близких по постановке задачи к настоящей работе. Так, в работе [9] рассматривался частный случай центральных потенциалов $V$ с особенностями типа обратных степеней. В силу центральности потенциала задача сводится к изучению обыкновенных дифференциальных операторов, для которых были найдены все самосопряженные расширения и тем самым построены искомые операторы с точечным взаимодействием. В работе [10] сначала рассматривался трехмерный оператор $H$, суженный на множество функций, исчезающих в окрестности нуля. Было показано, что в согласии с общей теорией любое самосопряженное расширение оператора $H$ является оператором того же вида с областью определения, которая состоит из функций

$$
f(\boldsymbol{r})=g(\boldsymbol{r})+c\left[G(\boldsymbol{r}, 0, i)-e^{i \alpha} G(\boldsymbol{r}, 0,-i)\right] .
$$

Здесь константа $c \in \mathbb{C}$, a $g$ является функцией из области определения оператора $-\Delta$, удовлетворяющей условию $g(0)=0$. Через $G\left(\boldsymbol{r}, \boldsymbol{r}^{\prime}, z\right)$ обозначена функция Грина оператора Шредингера с потенциалом $V$, определяемая как ядро оператора $G(z)=(H-z)^{-1}$, где $z \in \mathbb{C} \backslash \sigma(H)$ и $\sigma(H)$ - спектр гамильтониана $H$. Это обозначение для функции Грина будет использоваться везде далее. Константа $\alpha \in[0,2 \pi)$ является параметром оператора $\widetilde{H}$. По существу условие $(2)$ определяет поведение функций $f$ в точках, в которых потенциал $V$ имеет сингулярность. В рассматриваемом случае оно позволяет установить вид граничного условия при $r=0$, которому должна удовлетворять собственная функция оператора Шредингера. Поскольку функция $g$ из (2) не дает вклада при $r=0$, для получения граничного условия нужно исследовать асимптотическое поведение функции Грина $G(\boldsymbol{r}, 0, z)$ при $r \rightarrow 0$. Одной из целей настоящей работы является исследование этой асимптотики в случае определенного класса потенциалов $V$ с особенностями в нуле. Этот класс введен в начале раздела 2 настоящей работы. 
Существует другой подход, который позволяет добавлять точечный потенциал в уравнение Шредингера с гамильтонианом $H$, основанный на использовании так называемого псевдопотенциала. Псевдопотенциал определяется с помощью функционала, действующего на собственную функцию. Этот подход применялся в работе [11] для определения функции Грина свободного гамильтониана $-\Delta$, возмущенного точечным взаимодействием. В настоящей работе мы показываем, как можно использовать метод псевдопотенциала для получения явного вида гамильтониана $\widetilde{H}$. Как и в методе, основанном на самосопряженных расширениях, определяющее значение имеет асимптотика функции Грина $G(\boldsymbol{r}, 0, z)$ при $\boldsymbol{r} \rightarrow 0$.

Рассматриваемые гамильтонианы имеют ряд физических приложений. Среди недавних примеров использования точечного взаимодействия отметим применение к задаче рассеяния позитрона на атоме водорода, где для описания взаимодействия между позитроном и атомом использовался модельный гамильтониан с точечным потенциалом с мнимой константой связи $\lambda$. Последнее необходимо для описания процесса аннигиляции позитрона и электрона в рамках нерелятивистской квантовой механики [12], [13]. При этом кулоновское взаимодействие актуально лишь для малых расстояний, тогда как асимптотически при больших расстояниях между электроном и атомом взаимодействие становится короткодействующим. Отметим, что постановка задачи о поиске самосопряженного расширения оператора $H$ имеет смысл только в случае вещественной константы связи $\lambda$. В отличие от метода самосопряженных расширений в подходе с псевдопотенциалом принадлежность константы связи $\lambda$ полю вещественных чисел не требуется. В частности и по этой причине, в настоящей работе реализован подход, допускающий комплексные значения константы связи точечного потенциала, примененный ранее в работе авторов [14] для суперпозиции кулоновского и точечного потенциалов.

Работа организована следующим образом. В разделе 2 исследуется функция Грина оператора Шредингера с потенциалом, имеющим степенную особенность. В разделе 3 проведено построение псевдопотенциала. Вычисленная в разделе 2 асимптотика функции Грина используется для определения вида псевдопотенциала. Раздел 4 содержит заключение.

Условимся о некоторых обозначениях, применяющихся на протяжении работы. Жирными буквами обозначаются векторы из $\mathbb{R}^{3}$, скалярное произведение векторов записывается в виде $\boldsymbol{r} \cdot \boldsymbol{q}$, обычным шрифтом обозначаются скалярные величины и, в частности, модули векторов, например $r=|\boldsymbol{r}|$, при этом единичные векторы обозначаются как $\hat{\boldsymbol{r}}=\boldsymbol{r} / r$.

\section{2. ОСОБЕННОСТИ ФУНКЦИИ ГРИНА ОПЕРАТОРА ШРЕДИНГЕРА С ПОТЕНЦИАЛОМ, ИМЕЮЩИМ СТЕПЕННУЮ ОСОБЕННОСТЬ}

Прежде всего введем класс локальных потенциалов $V(\boldsymbol{r})$, для которых будут получены результаты настоящей работы. Предположим, что потенциал $V(\boldsymbol{r})$ является вещественнозначной гладкой функцией при всех $r=|\boldsymbol{r}|>0$, удовлетворяющей следующему условию: для всех $\boldsymbol{r}$, кроме малой окрестности точки $\boldsymbol{r}=0$, существует 
константа $M>0$ такая, что выполнено неравенство

$$
|V(\boldsymbol{r})| \leqslant M(1+r)^{-1-\delta}, \quad \delta>1 .
$$

Существенным для нашего рассмотрения является сингулярное поведение потенциала $V$ в окрестности точки $\boldsymbol{r}=0$. А именно, предполагается, что при $r \rightarrow 0$ потенциал может быть представлен в виде

$$
V(\boldsymbol{r})=r^{-\rho} Q(\boldsymbol{r})
$$

где функция $Q(\boldsymbol{r})$ является непрерывной вместе со своими производными до второго порядка и, соответственно, имеет конечный предел

$$
\lim _{r \rightarrow 0} Q(\boldsymbol{r})=V_{0}
$$

В настоящей работе для показателя $\rho$ будет использоваться ограничение $\rho<3 / 2$. Описанный класс потенциалов будем обозначать как $\mathfrak{V}(\rho, \delta)$.

Функция Грина оператора Шредингера $H$ является решением неоднородного уравнения

$$
[-\Delta+V(\boldsymbol{r})-z] G\left(\boldsymbol{r}, \boldsymbol{r}^{\prime}, z\right)=\delta\left(\boldsymbol{r}-\boldsymbol{r}^{\prime}\right)
$$

и также удовлетворяет интегральному уравнению Липпмана-Швингера

$$
G\left(\boldsymbol{r}, \boldsymbol{r}^{\prime}, z\right)=G_{0}\left(\boldsymbol{r}, \boldsymbol{r}^{\prime}, z\right)-\int d \boldsymbol{q} G_{0}(\boldsymbol{r}, \boldsymbol{q}, z) V(\boldsymbol{q}) G\left(\boldsymbol{q}, \boldsymbol{r}^{\prime}, z\right)
$$

Здесь $G_{0}-$ функция Грина оператора $-\Delta$ свободного движения в координатном представлении, которая имеет вид

$$
G_{0}\left(\boldsymbol{r}, \boldsymbol{r}^{\prime}, z\right)=\frac{1}{4 \pi} \frac{e^{i \sqrt{z}\left|\boldsymbol{r}-\boldsymbol{r}^{\prime}\right|}}{\left|\boldsymbol{r}-\boldsymbol{r}^{\prime}\right|}
$$

где $\operatorname{Im} \sqrt{z} \geqslant 0$. В дальнейшем нам потребуется асимптотическое поведение при $r \rightarrow 0$ функции $G(\boldsymbol{r}, 0, z)$. Обозначив $g(\boldsymbol{r}, z)=G(\boldsymbol{r}, 0, z)$ и положив $r^{\prime}=0$ в (6), придем к уравнению

$$
g(\boldsymbol{r}, z)=G_{0}(\boldsymbol{r}, 0, z)-\int d \boldsymbol{q} G_{0}(\boldsymbol{r}, \boldsymbol{q}, z) V(\boldsymbol{q}) g(\boldsymbol{q}, z),
$$

которое, как и в работе [15], является нашим основным средством исследования. В этом разделе мы покажем, что уравнение (8) в случае потенциалов $V \in \mathfrak{V}(\rho, \delta)$ имеет единственное решение, и опишем особенности этого решения.

Введем множество $\mathfrak{C}(\beta)$ непрерывных функций $f(\boldsymbol{r})$, удовлетворяющих условию, что для любой $f(\boldsymbol{r}) \in \mathfrak{C}(\beta)$ существует константа $C_{f}$ такая, что

$$
|f(\boldsymbol{r})| \leqslant C_{f}(1+r)^{-\beta}
$$

Хорошо известно, что множество $\mathfrak{C}(\beta)$ превращается в банахово пространство после введения нормы

$$
\sup (1+r)^{\beta}|f(\boldsymbol{r})|=\|f\|_{\beta} .
$$


Покажем, что интегральный оператор с ядром

$$
G_{0}(\boldsymbol{r}, \boldsymbol{q}, z) V(\boldsymbol{q})
$$

из правой части уравнения (8) является компактным в $\mathfrak{C}(\beta)$ при $0 \leqslant \beta<1$. Для этого рассмотрим интеграл

$$
\Phi(\boldsymbol{r})=\int d \boldsymbol{q} \frac{e^{i \sqrt{z}|\boldsymbol{r}-\boldsymbol{q}|}}{|\boldsymbol{r}-\boldsymbol{q}|} V(\boldsymbol{q}) f(\boldsymbol{q}),
$$

в котором $V \in \mathfrak{V}(\rho, \delta), f \in \mathfrak{C}(\beta)$ и $\operatorname{Im} \sqrt{z} \geqslant 0$. Покажем, что функция $\Phi \in \mathfrak{C}\left(\beta^{\prime}\right)$ с $\beta^{\prime}>\beta$. Отсюда стандартным образом будет следовать, что ядро (10) определяет в $\mathfrak{C}(\beta)$ компактный оператор.

Чтобы разделить вклады подынтегрального выражения (11) в начале координат и на бесконечности, разобьем область интегрирования на две части $\Omega_{j} \subset \mathbb{R}^{3}, j=1,2$, заданные как

$$
\Omega_{1}=\left\{\boldsymbol{q}: q<r_{0}\right\}, \quad \Omega_{2}=\left\{\boldsymbol{q}: q>r_{0}\right\} .
$$

Радиус $r_{0}<1$ выбирается настолько малым, чтобы в области $\Omega_{1}$ можно было использовать представление (4) для $V(\boldsymbol{r})$. Тогда интеграл (11) представляется в виде суммы интегралов, $\Phi=\Phi_{1}+\Phi_{2}$, где

$$
\Phi_{j}(\boldsymbol{r})=\int_{\Omega_{j}} d \boldsymbol{q} \frac{e^{i \sqrt{z}|\boldsymbol{r}-\boldsymbol{q}|}}{|\boldsymbol{r}-\boldsymbol{q}|} V(\boldsymbol{q}) f(\boldsymbol{q}), \quad j=1,2 .
$$

Оценим сначала интеграл $\Phi_{1}$ по абсолютной величине. В силу того что $\operatorname{Im} \sqrt{z} \geqslant 0$, имеем $\left|e^{i \sqrt{z}|\boldsymbol{r}-\boldsymbol{q}|}\right|=e^{-\operatorname{Im} \sqrt{z}|\boldsymbol{r}-\boldsymbol{q}|} \leqslant 1$ и, следовательно,

$$
\left|\Phi_{1}(\boldsymbol{r})\right| \leqslant \int_{\Omega_{1}} d \boldsymbol{q} \frac{1}{|\boldsymbol{r}-\boldsymbol{q}|}|Q(\boldsymbol{q})| \frac{C_{f}}{q^{\rho}(1+q)^{\beta}},
$$

где также было использовано представление (4) для потенциала $V$ и условие (9) для $f$. Покажем, что правая часть последнего выражения ограничена. Действительно, в силу ограниченности $Q$ в области интегрирования найдется константа $C_{Q}$ такая, что $|Q| \leqslant C_{Q}$, и, следовательно,

$$
\int_{\Omega_{1}} d \boldsymbol{q} \frac{1}{|\boldsymbol{r}-\boldsymbol{q}|}|Q(\boldsymbol{q})| \frac{1}{q^{\rho}(1+q)^{\beta}} \leqslant C_{Q} \int_{\Omega_{1}} d \boldsymbol{q} \frac{1}{|\boldsymbol{r}-\boldsymbol{q}|} q^{-\rho} .
$$

Вычисление последнего интеграла легко выполняется при помощи формулы

$$
\frac{1}{\left|\boldsymbol{q}-\boldsymbol{q}^{\prime}\right|}=\frac{1}{q_{>}} \sum_{\ell=0}^{\infty} \frac{q_{<}^{\ell}}{q_{>}^{\ell}} P_{\ell}\left(\hat{\boldsymbol{q}} \cdot \hat{\boldsymbol{q}}^{\prime}\right)
$$

где $P_{\ell}$ - полином Лежандра и, как обычно, $q_{>}=\max \left\{q, q^{\prime}\right\}, q_{<}=\min \left\{q, q^{\prime}\right\}$. Используя ортогональность полиномов Лежандра, мы без труда убеждаемся в справедливости формулы

$$
\int d \hat{\boldsymbol{q}} \frac{1}{|\boldsymbol{r}-\boldsymbol{q}|}=\frac{2 \pi}{r q}(r+q-|r-q|)
$$


Подставляя это равенство в правую часть формулы (13), при $r \leqslant r_{0}$ мы приходим к результату

$$
\begin{aligned}
\int_{\Omega_{1}} d \boldsymbol{q} \frac{1}{|\boldsymbol{r}-\boldsymbol{q}|} q^{-\rho} & =\frac{4 \pi}{r} \int_{0}^{r} d q q^{2-\rho}+4 \pi \int_{r}^{r_{0}} d q q^{1-\rho}= \\
& =\frac{4 \pi r_{0}^{2-\rho}}{2-\rho}-\frac{4 \pi r^{2-\rho}}{(3-\rho)(2-\rho)}
\end{aligned}
$$

Последнее выражение и, следовательно, интеграл $\Phi_{1}$ ограничены при $r \leqslant r_{0}$.

В случае $r>r_{0}$ имеем

$$
\int_{\Omega_{1}} d \boldsymbol{q} \frac{1}{|\boldsymbol{r}-\boldsymbol{q}|} q^{-\rho}=\frac{4 \pi}{r} \int_{0}^{r_{0}} d q q^{2-\rho}=\frac{4 \pi}{r} \frac{r_{0}^{3-\rho}}{3-\rho}
$$

и тогда приходим к оценке

$$
\left|\Phi_{1}(\boldsymbol{r})\right| \leqslant C_{f} C_{Q} \frac{4 \pi}{r} \frac{r_{0}^{3-\rho}}{3-\rho} .
$$

Собирая полученные результаты, приходим к заключению, что существует константа $C_{1}$ такая, что для любых значений $r$ имеет место оценка

$$
\left|\Phi_{1}(\boldsymbol{r})\right| \leqslant C_{1} \frac{1}{1+r}
$$

Перейдем к изучению интеграла $\Phi_{2}$. Оценим этот интеграл по абсолютному значению. Имеем с учетом (3) и (9)

$$
\left|\Phi_{2}(\boldsymbol{r})\right| \leqslant C_{f} M \int_{r_{0}}^{+\infty} d q q^{2} \frac{1}{(1+q)^{1+\beta+\delta}} \int d \hat{\boldsymbol{q}} \frac{1}{|\boldsymbol{r}-\boldsymbol{q}|} .
$$

Рассмотрим сначала случай ограниченных $r \leqslant r_{0}$. В этом случае в силу формулы (14)

$$
\left|\Phi_{2}(\boldsymbol{r})\right| \leqslant 4 \pi C_{f} M \int_{r_{0}}^{+\infty} d q \frac{q}{(1+q)^{1+\beta+\delta}} .
$$

Поскольку последний интеграл сходится и не зависит от $r$, интеграл $\Phi_{2}$ ограничен при $r \leqslant r_{0}$.

Рассмотрим теперь случай неограниченных сверху $r>r_{0}$. Для таких $r$, используя равенство (14) для интеграла по угловой части переменной $\boldsymbol{q}$, получаем в (17)

$$
\left|\Phi_{2}(r)\right| \leqslant \frac{2 \pi C_{f} M}{r} \int_{r_{0}}^{+\infty} d q \frac{q(r+q-|r-q|)}{(1+q)^{1+\beta+\delta}} .
$$

Замена переменной $q=r \tau$ в интеграле правой части (18) позволяет получить неравенство

$$
\begin{aligned}
\frac{2 \pi C_{f} M}{r^{\beta+\delta-1}} & \int_{r_{0} / r}^{+\infty} d \tau \frac{\tau}{(1 / r+\tau)^{1+\beta+\delta}}(1+\tau-|1-\tau|) \leqslant \\
& \leqslant \frac{2 \pi C_{f} M}{r^{\beta+\delta-1}} \int_{0}^{+\infty} d \tau \frac{1}{\tau^{\beta+\delta}}(1+\tau-|1-\tau|)
\end{aligned}
$$


Чтобы показать, что последний интеграл сходится, разобьем область интегрирования по переменной $\tau$ на два интервала $\tau \geqslant 1$ и $0 \leqslant \tau<1$. На этих интервалах выражение $1+\tau-|1-\tau|$ равняется соответственно 2 и $2 \tau$, следовательно, для интеграла из правой части (19) мы получаем

$$
\int_{0}^{+\infty} d \tau \frac{1}{\tau^{1+\beta+\delta}}(1+\tau-|1-\tau|)=2 \int_{0}^{1} d \tau \tau^{1-\beta-\delta}+2 \int_{1}^{+\infty} d \tau \frac{1}{\tau^{\beta+\delta}} .
$$

Поскольку $0 \leqslant \beta<1$, а показатель $\delta>1$ можно считать сколь угодно близким к единице, оба интеграла в правой части последнего равенства сходятся. В результате приведенного анализа правой части (19) при $r>r_{0}$ можно написать оценку

$$
\left|\Phi_{2}(\boldsymbol{r})\right| \leqslant \frac{C_{\Phi}}{r^{\beta+\delta-1}}
$$

с некоторой константой $C_{\Phi}$.

Объединяя полученные оценки, приходим к заключению, что для всех $r$ доказана справедливость неравенства

$$
\left|\Phi_{2}(\boldsymbol{r})\right| \leqslant C_{2} \frac{1}{(1+r)^{\beta+\delta-1}} .
$$

Теперь можно объединить результаты (16) и (20) в окончательную оценку абсолютного значения интеграла $\Phi$ :

$$
|\Phi(\boldsymbol{r})| \leqslant\left|\Phi_{1}(\boldsymbol{r})\right|+\left|\Phi_{2}(\boldsymbol{r})\right| \leqslant C \frac{1}{(1+r)^{\beta^{\prime}}},
$$

где $\beta^{\prime}=\min (1, \beta+\delta-1)$ и $C$ - некоторая константа. Поскольку $\beta+\delta-1>\beta$, из оценки (21) следует утверждение, сформулированное в начале данного раздела, а именно, при $0 \leqslant \beta<1$ ядро (10) интегрального уравнения (8) действительно определяет оператор, компактный в пространстве $\mathfrak{C}(\beta)$ (можно взять, например, $\beta=1 / 2)$.

Свободный член уравнения (8) как функция переменной $\boldsymbol{r}$ не принадлежит классу $\mathfrak{C}(\beta)$, поскольку имеет сингулярность при $r \rightarrow 0$. Действительно, асимптотическое поведение при $r \rightarrow 0$ свободного члена уравнения (8) следует из явного вида (7) функции Грина:

$$
G_{0}(\boldsymbol{r}, 0, z)=\frac{1}{4 \pi r}+\frac{i \sqrt{z}}{4 \pi}+O(r) .
$$

Чтобы избавиться от сингулярности в уравнении (8) при $r \rightarrow 0$, итерируем это уравнение один раз, что приводит к выражению

$$
\begin{aligned}
g(\boldsymbol{r}, z)= & G_{0}(\boldsymbol{r}, 0, z)-\int d \boldsymbol{q} G_{0}(\boldsymbol{r}, \boldsymbol{q}, z) V(\boldsymbol{q}) G_{0}(\boldsymbol{q}, 0, z)+ \\
& +\int d \boldsymbol{q} G_{0}(\boldsymbol{r}, \boldsymbol{q}, z) V(\boldsymbol{q}) \int d \boldsymbol{q}^{\prime} G_{0}\left(\boldsymbol{q}, \boldsymbol{q}^{\prime}, z\right) V\left(\boldsymbol{q}^{\prime}\right) g\left(\boldsymbol{q}^{\prime}, z\right) .
\end{aligned}
$$

Исследуем асимптотическое поведение второго слагаемого в правой части последнего равенства. Рассмотрим функцию

$$
g^{(1)}(\boldsymbol{r}, z)=\int d \boldsymbol{q} G_{0}(\boldsymbol{r}, \boldsymbol{q}, z) V(\boldsymbol{q}) G_{0}(\boldsymbol{q}, 0, z) .
$$


Вновь разобьем область интегрирования на две части $\Omega_{1,2}$, определенные в (12) при оценке интеграла $\Phi$, с теми же предположениями относительно радиуса $r_{0}$. Снова интеграл в правой части (22) записывается в виде суммы интегралов $I_{1,2}$, определенных формулами

$$
I_{j}(\boldsymbol{r})=\int_{\Omega_{j}} d \boldsymbol{q} G_{0}(\boldsymbol{r}, \boldsymbol{q}, z) V(\boldsymbol{q}) G_{0}(\boldsymbol{q}, 0, z) .
$$

Рассмотрим интеграл $I_{1}(\boldsymbol{r})$ при $r \leqslant r_{0}$. Выясним поведение данного интеграла при $r \rightarrow 0$. Выделим в подынтегральных сомножителях, входящих в интеграл $I_{1}$, их главные особенности, т. е. воспользуемся представлениями

$$
\begin{aligned}
G_{0}(\boldsymbol{r}, \boldsymbol{q}, z) & =\frac{1}{4 \pi|\boldsymbol{r}-\boldsymbol{q}|}+\frac{e^{i \sqrt{z}|\boldsymbol{r}-\boldsymbol{q}|}-1}{4 \pi|\boldsymbol{r}-\boldsymbol{q}|}, \\
V(\boldsymbol{q}) & =q^{-\rho} Q(\boldsymbol{q})=q^{-\rho} V_{0}+q^{-\rho}\left[Q(\boldsymbol{q})-V_{0}\right], \\
G_{0}(\boldsymbol{q}, 0, z) & =\frac{1}{4 \pi q}+\frac{e^{i \sqrt{z} q}-1}{4 \pi q} .
\end{aligned}
$$

С учетом свойств функции $Q$ второе слагаемое в $(25)$ можно записать в виде

$$
q^{-\rho}\left[Q(\boldsymbol{q})-V_{0}\right]=q^{-\rho+1} Q_{1}(\boldsymbol{q})
$$

где $Q_{1}$ является гладкой ограниченной функцией своих аргументов. Тогда наиболее сингулярный член $I_{1}(\boldsymbol{r})$ при $r \rightarrow 0$ будет даваться выражением, которое получается подстановкой в формулу (23) для $j=1$ главных членов разложений подынтегральных сомножителей, определенных в (24)-(27). Обозначим этот член через $I_{1}^{\mathrm{s}}$, его явный вид дается интегралом

$$
I_{1}^{\mathrm{S}}(r)=\frac{V_{0}}{(4 \pi)^{2}} \int_{\Omega_{1}} d \boldsymbol{q}|\boldsymbol{r}-\boldsymbol{q}|^{-1} q^{-\rho-1} .
$$

Вычисление интеграла по угловым переменным легко выполняется при помощи формулы (14) и приводит к результату

$$
I_{1}^{\mathrm{s}}(r)=\frac{V_{0}}{4 \pi}\left(\frac{1}{r} \int_{0}^{r} d q q^{1-\rho}+\int_{r}^{r_{0}} d q q^{-\rho}\right) .
$$

Далее в зависимости от величины $\rho$ получаем два случая: если $\rho \neq 1$, то

$$
I_{1}^{\mathrm{s}}(r)=\frac{V_{0}}{4 \pi(2-\rho)(\rho-1)} r^{-\rho+1}+\frac{V_{0}}{4 \pi(1-\rho)} r_{0}^{-\rho+1},
$$

если же $\rho=1$, то

$$
I_{1}^{\mathrm{s}}(r)=-\frac{V_{0}}{4 \pi} \ln r+\frac{V_{0}}{4 \pi}\left(1+\ln r_{0}\right)
$$

Из формулы $(29)$ видно, что если $1<\rho<3 / 2$, то $I_{1}^{\mathrm{s}}$ имеет полярную сингулярность вида $r^{-\rho+1}$; если же $\rho<1$, то первое слагаемое в $(29)$ исчезает при $r \rightarrow 0$, в этом случае интеграл $I_{1}^{\mathrm{s}}$ регулярен и имеет конечный предел.

4 Теоретическая и математическая физика, т. 183, №1, 2015 г. 
Из приведенного анализа интеграла (28) ясно, что учет менее сингулярных членов разложений подынтегральных функций в $I_{1}(\boldsymbol{r})$ дает несингулярные вклады при $r \rightarrow 0$. Действительно, покажем, что абсолютная величина остатка $I_{1}(\boldsymbol{r})-I_{1}^{\mathrm{s}}(r)$ ограничена по $r$ при $r \leqslant r_{0}$. Воспользуемся элементарной формулой

$$
(A+a)(B+b)(C+c)-a b c=A B C+a B(C+c)+A(B+b) c+(A+a) b C,
$$

где $A, B, C, a, b, c$ - комплексные числа. Применяя ее к произведению подынтегральных сомножителей в интеграле $I_{1}$, записанных в форме $(24)-(27)$, получим

$$
I_{1}(\boldsymbol{r})-I_{1}^{\mathrm{s}}(r)=I_{1}^{(1)}(\boldsymbol{r})+I_{1}^{(2)}(\boldsymbol{r})+I_{1}^{(3)}(\boldsymbol{r})+I_{1}^{(4)}(\boldsymbol{r}),
$$

где введены обозначения

$$
\begin{aligned}
& I_{1}^{(1)}(\boldsymbol{r})=\frac{1}{(4 \pi)^{2}} \int_{\Omega_{1}} d \boldsymbol{q} q^{-\rho} \frac{\left(e^{i \sqrt{z}|\boldsymbol{r}-\boldsymbol{q}|}-1\right)\left(e^{i \sqrt{z} q}-1\right)}{|\boldsymbol{r}-\boldsymbol{q}|} Q_{1}(\boldsymbol{q}), \\
& I_{1}^{(2)}(\boldsymbol{r})=\frac{1}{(4 \pi)^{2}} \int_{\Omega_{1}} d \boldsymbol{q} q^{-\rho} \frac{e^{i \sqrt{z} q}}{|\boldsymbol{r}-\boldsymbol{q}|} Q_{1}(\boldsymbol{q}), \\
& I_{1}^{(3)}(\boldsymbol{r})=\frac{1}{(4 \pi)^{2}} \int_{\Omega_{1}} d \boldsymbol{q} q^{-\rho-1} \frac{\left(e^{i \sqrt{z}|\boldsymbol{r}-\boldsymbol{q}|}-1\right)}{|\boldsymbol{r}-\boldsymbol{q}|} Q(\boldsymbol{q}), \\
& I_{1}^{(4)}(\boldsymbol{r})=\frac{V_{0}}{(4 \pi)^{2}} \int_{\Omega_{1}} d \boldsymbol{q} q^{-\rho-1} \frac{e^{i \sqrt{z}|\boldsymbol{r}-\boldsymbol{q}|}\left(e^{i \sqrt{z} q}-1\right)}{|\boldsymbol{r}-\boldsymbol{q}|} .
\end{aligned}
$$

Воспользуемся следующей легко проверяемой оценкой (см. монографию [16], формула 12.6):

$$
|\sin (k x)| \leqslant D e^{\left|k^{\prime}\right| x} \frac{|k| x}{1+|k| x},
$$

где $k^{\prime} \equiv \operatorname{Im} k$ и $D-$ константа. Это оценка справедлива для всех значений $k$ и для всех действительных $x \geqslant 0$. Теперь можно написать

$$
\left|e^{i \sqrt{z}|\boldsymbol{r}-\boldsymbol{q}|}-1\right|=2\left|e^{i \sqrt{z}|\boldsymbol{r}-\boldsymbol{q}| / 2}\right|\left|\sin \frac{\sqrt{z}|\boldsymbol{r}-\boldsymbol{q}|}{2}\right| \leqslant D e^{|\operatorname{Im} \sqrt{z}||\boldsymbol{r}-\boldsymbol{q}| / 2} \frac{|\sqrt{z}||\boldsymbol{r}-\boldsymbol{q}|}{1+|\sqrt{z}||\boldsymbol{r}-\boldsymbol{q}| / 2} .
$$

Поскольку $q, r \leqslant r_{0}$, имеем $|\boldsymbol{r}-\boldsymbol{q}| \leqslant 2 r_{0}$, следовательно, на рассматриваемом промежутке интегрирования

$$
\left|e^{i \sqrt{z}|\boldsymbol{r}-\boldsymbol{q}|}-1\right| \leqslant D|\sqrt{z}| e^{2 r_{0} \mid \operatorname{Im} \sqrt{z}}|\boldsymbol{r}-\boldsymbol{q}| \equiv D^{\prime}|\boldsymbol{r}-\boldsymbol{q}|
$$

при $r \leqslant r_{0}$, в том числе при $r=0$. С учетом оценки $\left|Q_{1}\right| \leqslant C^{\prime}$ и неравенства $(31)$ получаем

$$
\left|I_{1}^{(1)}(\boldsymbol{r})\right| \leqslant \frac{C^{\prime} D^{\prime 2}}{4 \pi} \int_{0}^{r_{0}} d q q^{3-\rho}=\frac{C^{\prime} D^{\prime 2} r_{0}^{4-\rho}}{4 \pi(4-\rho)}
$$

Отсюда следует, что интеграл $I_{1}^{(1)}$ ограничен при $r \leqslant r_{0}$. 
В случае интеграла $I_{1}^{(2)}$ имеем

$$
\left|I_{1}^{(2)}(\boldsymbol{r})\right| \leqslant \frac{C^{\prime} D^{\prime}}{(4 \pi)^{2}} \int_{\Omega_{1}} d \boldsymbol{q} \frac{q^{-\rho}}{|\boldsymbol{r}-\boldsymbol{q}|}=\frac{C^{\prime} D^{\prime}}{(4 \pi)^{2}}\left(\frac{4 \pi r_{0}^{2-\rho}}{2-\rho}-\frac{4 \pi r^{2-\rho}}{(3-\rho)(2-\rho)}\right),
$$

где мы воспользовались формулой (15). Из (32) также следует ограниченность интеграла при $r \leqslant r_{0}$.

Ограниченность при $r \leqslant r_{0}$ интегралов $I_{1}^{(3)}(\boldsymbol{r})$ и $I_{1}^{(4)}(\boldsymbol{r})$ доказывается аналогично. Отсюда следует утверждение, сделанное выше, об ограниченности при $r \leqslant r_{0}$ абсолютной величины остатка $I_{1}(\boldsymbol{r})-I_{1}^{\mathrm{s}}(r)$.

Продолжим исследование интеграла $I_{1}(\boldsymbol{r})$. Покажем, что при $r>r_{0}$ интеграл $I_{1}(\boldsymbol{r})$ допускает оценку типа (9) с $\beta=1$. Действительно, оценивая его по абсолютной величине и выполняя интегрирование по угловой части переменной $\boldsymbol{q}$ при помощи формулы (14), с учетом $|Q| \leqslant C_{Q}$ приходим к оценке

$$
\left|I_{1}(\boldsymbol{r})\right| \leqslant \frac{4 \pi C_{Q}}{r} \int_{0}^{r_{0}} d q q^{1-\rho}
$$

где последний интеграл сходится.

Рассмотрим наконец интеграл $I_{2}(\boldsymbol{r})$. Так как в области $\Omega_{2}$ функция $G_{0}(\boldsymbol{q}, 0, z)$ допускает оценку типа (9) с $\beta=1$, интеграл $I_{2}(\boldsymbol{r})$ также допускает эту оценку, что следует из анализа интеграла $\Phi_{2}$. Из проведенных рассмотрений вытекает окончательное утверждение относительно поведения первой итерации $g^{(1)}(\boldsymbol{r}, z)$, которое нам понадобится в дальнейшем: при $r \leqslant r_{0}$ функция $g^{(1)}$ имеет сингулярное поведение, которое описывается формулами (29), (30), при этом существует такая константа $B$, что

$$
\left|g^{(1)}(\boldsymbol{r}, z)\right| \leqslant B g_{\mathrm{s}}^{(1)}(r, z),
$$

где сингулярная функция $g_{\mathrm{s}}^{(1)}$ в соответствии с формулами $(29),(30)$ имеет вид

$$
g_{\mathrm{s}}^{(1)}(r, z)=\left\{\begin{array}{cll}
1 & \text { при } & \rho<1, \\
-\ln r & \text { при } & \rho=1, \\
r^{-\rho+1} & \text { при } & 1<\rho<3 / 2 .
\end{array}\right.
$$

При $r>r_{0}$ первая итерация $g^{(1)}(\boldsymbol{r}, z)$ допускает оценку типа $(9)$ с $\beta=1$.

Итак, первая итерация уравнения (8) имеет сингулярность при $r \rightarrow 0$ и не принадлежит классу $\mathfrak{C}(\beta)$. Покажем, что следующая итерация

$$
g^{(2)}(\boldsymbol{r}, z) \equiv \int d \boldsymbol{q} G_{0}(\boldsymbol{r}, \boldsymbol{q}, z) V(\boldsymbol{q}) \int d \boldsymbol{q}^{\prime} G_{0}\left(\boldsymbol{q}, \boldsymbol{q}^{\prime}, z\right) V\left(\boldsymbol{q}^{\prime}\right) G_{0}\left(\boldsymbol{q}^{\prime}, 0, z\right)
$$

принадлежит классу $\mathfrak{C}(\beta)$. Внутренний интеграл по $\boldsymbol{q}^{\prime}$ представляет собой функцию $g^{(1)}(\boldsymbol{q}, z)$, исследованную выше. Как функция от $\boldsymbol{q}$ он может иметь сингулярность не сильнее чем $q^{-\rho+1}$ (или $\ln q$ в случае $\rho=1$ ) при $q \leqslant r_{0}$ и допускает оценку типа (9) с $\beta=1$ при $q>r_{0}$. Вновь разобъем внешний интеграл по $\boldsymbol{q}$ на интегралы по областям $\Omega_{1}$ и $\Omega_{2}$. Полученные таким образом интегралы имеют вид

$$
J_{j}(\boldsymbol{r})=\int_{\Omega_{j}} d \boldsymbol{q} G_{0}(\boldsymbol{r}, \boldsymbol{q}, z) V(\boldsymbol{q}) g^{(1)}(\boldsymbol{q}, z) .
$$


Оценим интеграл $J_{1}$ по абсолютной величине. При этом будем различать случаи $\rho<1, \rho=1$ и $1<\rho<3 / 2$. Рассмотрим вначале случай ограниченных $r \leqslant r_{0}$. В соответствии с формулами (33) и (34) в случае $1<\rho<3 / 2$ будем иметь

$$
\begin{aligned}
\left|J_{1}(\boldsymbol{r})\right| & \leqslant \frac{B C_{Q}}{4 \pi} \int_{\Omega_{1}} d \boldsymbol{q} \frac{q^{1-2 \rho}}{|\boldsymbol{r}-\boldsymbol{q}|}=B C_{Q}\left(\frac{1}{r} \int_{0}^{r} d q q^{3-2 \rho}+\int_{r}^{r_{0}} d q q^{2-2 \rho}\right)= \\
& =B C_{Q}\left(-\frac{1}{2(2-\rho)(3-2 \rho)} r^{3-2 \rho}+\frac{1}{3-2 \rho} r_{0}^{3-2 \rho}\right) .
\end{aligned}
$$

Таким образом, с учетом того, что $\rho<3 / 2$, сингулярность при $r \rightarrow 0$ не возникает, и интеграл $J_{1}$ ограничен по абсолютной величине. Это утверждение является верным также и для случаев $\rho=1$ и $\rho<1$, которые рассматриваются аналогично.

Рассмотрим теперь случай неограниченных сверху $r>r_{0}$. При $1<\rho<3 / 2$ получаем

$$
\left|J_{1}(\boldsymbol{r})\right| \leqslant \frac{B C_{Q}}{4 \pi} \int_{\Omega_{1}} d \boldsymbol{q} \frac{q^{1-2 \rho}}{|\boldsymbol{r}-\boldsymbol{q}|}=\frac{B C_{Q}}{r} \int_{0}^{r_{0}} d q q^{3-2 \rho}=\frac{B C_{Q} r_{0}^{4-2 \rho}}{(4-2 \rho) r} .
$$

Аналогично можно показать, что такой же характер убывания на бесконечности имеет абсолютное значение интеграла $J_{1}(\boldsymbol{r})$ и при $\rho \leqslant 1$.

Интеграл $J_{2}$ по области $\Omega_{2}$ рассматривается совершенно аналогично интегралу $I_{2}$.

Итак, мы получаем, что вторая итерация $g^{(2)}$, определенная в $(35)$, принадлежит классу $\mathfrak{C}(\beta)$.

Если теперь ввести вместо $g(\boldsymbol{r}, z)$ функцию $\gamma(\boldsymbol{r}, z)$ по формуле

$$
\gamma(\boldsymbol{r}, z)=g(\boldsymbol{r}, z)-G_{0}(\boldsymbol{r}, 0, z)+g^{(1)}(\boldsymbol{r}, z),
$$

то эта функция будет удовлетворять интегральному уравнению

$$
\gamma(\boldsymbol{r}, z)=g^{(2)}(\boldsymbol{r}, z)-\int d \boldsymbol{q} G_{0}(\boldsymbol{r}, \boldsymbol{q}, z) V(\boldsymbol{q}) \gamma(\boldsymbol{q}, z) .
$$

Выше было показано, что свободный член $g^{(2)}$ принадлежит пространству $\mathfrak{C}(\beta)$, а ядро $G_{0}(\boldsymbol{r}, \boldsymbol{q}, z) V(\boldsymbol{q})$ определяет компактный в $\mathfrak{C}(\beta)$ оператор. Используя методику работы [17], нетрудно показать, что однородное уравнение (36) может иметь нетривиальное решение только для дискретного набора вещественных значений параметра $z$. Эти значения определяют дискретный спектр $\sigma_{\mathrm{d}}(H)$ оператора $H$. На основании альтернативы Фредгольма приходим тогда к заключению, что решение уравнения (36) существует и единственно при всех $z \notin \sigma_{\mathrm{d}}(H)$ и является функцией из класса $\mathfrak{C}(\beta)$.

Объединяя результаты, полученные в данном разделе, сформулируем окончательное утверждение о поведении функции $G(\boldsymbol{r}, 0, z)$ при $r \rightarrow 0$ : в случае $1<\rho<3 / 2$ имеет место представление

$$
G(\boldsymbol{r}, 0, z)=\frac{1}{4 \pi}\left(\frac{1}{r}+\frac{A_{0}}{r^{\rho-1}}\right)+B_{1}+o(1),
$$

в случае $\rho=1$ справедливо равенство

$$
G(\boldsymbol{r}, 0, z)=\frac{1}{4 \pi}\left(\frac{1}{r}+V_{0} \ln r\right)+B_{2}+o(1),
$$


а в случае $\rho<1$ поведение функции Грина имеет стандартный характер:

$$
G(\boldsymbol{r}, 0, z)=\frac{1}{4 \pi r}+B_{3}+o(1)
$$

Здесь константа $A_{0}$ дается выражением

$$
A_{0}=\frac{V_{0}}{(2-\rho)(1-\rho)},
$$

и все конечные вклады от соответствующих выражений из полученных выше формул обозначены как $B_{j}, j=1,2,3$. Следует отметить, что асимптотическое поведение функции Грина (38) при $\rho=1$ идентично соответствующей асимптотике кулоновской функции Грина [14].

\section{3. МЕТОД ПСЕВДОПОТЕНЦИАЛА}

В этом разделе мы опишем метод добавления точечного потенциала в уравнение Шредингера с гамильтонианом $H$ вида (1), основанный на использовании псевдопотенциала. Этот метод применялся, в частности, в работе [11] для определения функции Грина свободного гамильтониана, возмущенного точечным взаимодействием.

Рассмотрим уравнение Шредингера, в котором к невозмущенному гамильтониану $H$ добавлен член с псевдопотенциалом:

$$
\left[-\Delta+V(\boldsymbol{r})-k^{2}\right] \psi(\boldsymbol{r}, \boldsymbol{k})+\lambda W(\boldsymbol{r}) \psi(\boldsymbol{r}, \boldsymbol{k})=0
$$

Псевдопотенциал $W$ определяется равенством

$$
W(\boldsymbol{r}) \psi(\boldsymbol{r}, \boldsymbol{k})=\delta(\boldsymbol{r}) \beta
$$

где константа $\beta$ является значением некоторого линейного функционала от решения $\psi$. Определение вида этого функционала и составляет основную задачу данного раздела.

С учетом предыдущего равенства перепишем уравнение (40) в виде

$$
\left[-\Delta+V(\boldsymbol{r})-k^{2}\right] \psi(\boldsymbol{r}, \boldsymbol{k})+\lambda \delta(\boldsymbol{r}) \beta=0 .
$$

Предположим, что $k^{2} \notin \sigma_{\mathrm{d}}(H)$, тогда, обращая оператор в левой части предыдущего равенства, перейдем к интегральному уравнению Липпмана-Швингера

$$
\psi(\boldsymbol{r}, \boldsymbol{k})=\psi_{0}(\boldsymbol{r}, \boldsymbol{k})-\lambda \int d \boldsymbol{r}^{\prime} G\left(\boldsymbol{r}, \boldsymbol{r}^{\prime}, k^{2}+i 0\right) \delta\left(\boldsymbol{r}^{\prime}\right) \beta .
$$

Функция $\psi_{0}$ соответствует состоянию рассеяния и является решением уравнения

$$
\left[-\Delta+V(\boldsymbol{r})-k^{2}\right] \psi_{0}(\boldsymbol{r}, \boldsymbol{k})=0
$$

удовлетворяя при этом асимптотическому граничному условию

$$
\psi_{0}(\boldsymbol{r}, \boldsymbol{k}) \sim e^{i \boldsymbol{k} \cdot \boldsymbol{r}}+A r^{-1} e^{i k r}
$$


при $r \rightarrow \infty$. Выполняя интегрирование в (42) при помощи дельта-функции, приходим к представлению

$$
\psi(\boldsymbol{r}, \boldsymbol{k})=\psi_{0}(\boldsymbol{r}, \boldsymbol{k})-\lambda G\left(\boldsymbol{r}, 0, k^{2}+i 0\right) \beta
$$

Теперь можно выразить асимптотику при $r \rightarrow 0$ функции $\psi$, являющейся решением уравнения Шредингера (40) с псевдопотенциалом $\lambda W$, определенным формулой $(41)$, через константу $\beta$. Как будет показано ниже, предел функции $\psi_{0}$ при $r \rightarrow 0$ конечен в случае $V \in \mathfrak{V}(\rho, \delta)$, и тем самым нетривиальная часть асимптотики функции $\psi$ в нуле полностью определяется членом, содержащим функцию Грина в (43). В результате мы убедимся, что асимптотика функции Грина $G(\boldsymbol{r}, 0, z)$ при $r \rightarrow 0$ имеет определяющее значение при построении формализма точечного потенциала.

Поскольку асимптотическое поведение функции Грина $G(\boldsymbol{r}, 0, z)$ при $r \rightarrow 0$ было подробно исследовано в предыдущем разделе, нам остается исследовать поведение функции $\psi_{0}$ в нуле. Это можно сделать на основе следующего интегрального уравнения Липпмана-Швингера:

$$
\psi_{0}(\boldsymbol{r}, \boldsymbol{k})=e^{i \boldsymbol{k} \cdot \boldsymbol{r}}-\int d \boldsymbol{q} G_{0}\left(\boldsymbol{r}, \boldsymbol{q}, k^{2}+i 0\right) V(\boldsymbol{q}) \psi_{0}(\boldsymbol{q}, \boldsymbol{k}) .
$$

Хорошо известно [17], [15], что решение этого уравнения существует, выражается в терминах функции Грина формулой

$$
\psi_{0}(\boldsymbol{r}, \boldsymbol{k})=\lim _{\epsilon \rightarrow 0}\left(-i \epsilon \int d \boldsymbol{q} G\left(\boldsymbol{r}, \boldsymbol{q}, k^{2}+i \epsilon\right) e^{i \boldsymbol{q} \cdot \boldsymbol{k}}\right)
$$

и является ограниченной функцией своего первого аргумента. В последнем легко убедиться в нашем случае потенциалов из класса $\mathfrak{V}(\rho, \delta)$, фактически повторяя рассуждения предыдущего раздела при $\beta=0$. В результате можем заключить, что для потенциалов из класса $\mathfrak{V}(\rho, \delta)$ асимптотика функции $\psi_{0}(\boldsymbol{r}, \boldsymbol{k})$ регулярна при $r \rightarrow 0$ и эта функция имеет конечный предел в начале координат.

Сингулярное поведение полного решения $\psi$ тем самым полностью определяется сингулярностями функции Грина. В соответствии с формулами (37)-(39) мы имеем три случая для асимптотики волновой функции в зависимости от значения параметра потенциала $\rho$ : в случае наиболее сильной сингулярности потенциала при $1<\rho<3 / 2$ асимптотика функции $\psi$ имеет вид

$$
\psi(\boldsymbol{r}, \boldsymbol{k})=\frac{\alpha_{1}}{4 \pi}\left(\frac{1}{r}+\frac{A_{0}}{r^{\rho-1}}\right)+\beta_{1}+o(1),
$$

в случае $\rho=1$ она дается выражением

$$
\psi(\boldsymbol{r}, \boldsymbol{k})=\frac{\alpha_{2}}{4 \pi}\left(\frac{1}{r}+V_{0} \ln r\right)+\beta_{2}+o(1),
$$

а в случае $\rho<1$ верно равенство

$$
\psi(\boldsymbol{r}, \boldsymbol{k})=\frac{\alpha_{3}}{4 \pi r}+\beta_{3}+o(1) .
$$


Присутствующие в последних формулах константы определяются как

$$
A_{0}=\frac{V_{0}}{(2-\rho)(1-\rho)}, \quad \alpha_{j}=-\lambda \beta_{j}, \quad \beta_{j}=\psi_{0}(0, \boldsymbol{k})-\lambda B_{j} \beta_{j}, \quad j=1,2,3 .
$$

Заметим, что константы $\alpha_{j}$ и $\beta_{j}$ не являются независимыми. Единственным независимым параметром, определяющим вид точечного потенциала, является константа связи $\lambda=-\alpha_{j} / \beta_{j}$.

Теперь, следуя методу, описанному в работе [13], можно вычислить вид псевдопотенциала (41) из асимптотических выражений (44)-(46). Для всех трех случаев псевдопотенциал может быть записан в виде

$$
\lambda W_{j}(\boldsymbol{r})=\lambda \delta(\boldsymbol{r}) \frac{d}{d \omega_{j}} \omega_{j}=\lambda \delta(\boldsymbol{r})\left(1+\omega_{j} \frac{d}{d \omega_{j}}\right),
$$

где переменные $\omega_{j}$ определены формулами

$$
\frac{1}{\omega_{1}}=r^{-1}+A_{0} r^{-\rho+1}, \quad \frac{1}{\omega_{2}}=r^{-1}+V_{0} \ln r, \quad \frac{1}{\omega_{3}}=r^{-1} .
$$

Действительно, в новых переменных $\omega_{j}$ асимптотические выражения (44)-(46) переписываются в виде

$$
\psi(\boldsymbol{r}, \boldsymbol{k})=\frac{\alpha_{j}}{4 \pi \omega_{j}}+\beta_{j}+o(1)
$$

и, следовательно,

$$
\lambda \delta(\boldsymbol{r}) \frac{d}{d \omega_{j}}\left(\omega_{j} \psi(\boldsymbol{r}, \boldsymbol{k})\right)=\lambda \beta_{j} \delta(\boldsymbol{r}),
$$

что согласуется с определением псевдопотенциала (41) если положить $\beta=\beta_{j}$. Из последнего равенства получаем

$$
\beta_{j}=\lim _{r \rightarrow 0} \frac{d}{d \omega_{j}}\left(\omega_{j} \psi(\boldsymbol{r}, \boldsymbol{k})\right) .
$$

Вновь подчеркнем, что в случае $\rho=1$ построенный псевдопотенциал совпадает с псевдопотенциалом для чисто кулоновского случая [14]. Это завершает построение псевдопотенциала и тем самым оператора $\widetilde{H}$ в случае потенциалов $V \in \mathfrak{V}(\rho, \delta)$.

\section{4. ЗАКЛЮЧЕНИЕ}

В предложенной работе построены гамильтонианы с суперпозицией короткодействующего и точечного потенциалов. Рассмотрен случай, когда особенность короткодействующего потенциала попадает на носитель точечного взаимодействия. В результате этого точечное взаимодействие модифицируется. Основным техническим средством для нахождения этой модификации является интегральное уравнение для функции Грина.

В работе показано, что функция Грина $G(\boldsymbol{r}, 0, z)$ оператора Шредингера с потенциалом, имеющим в нуле сингулярность вида $O\left(r^{-\rho}\right)$, помимо стандартной особенности $1 / 4 \pi r$, обладает дополнительной сингулярностью вида $O\left(r^{-\rho+1}\right)$, за исключением случая $\rho=1$, в котором возникает логарифмическая сингулярность. Такие сингулярности и приводят к модифицированным потенциалам нулевого радиуса. 
Мы рассмотрели класс короткодействующих потенциалов $\mathfrak{V}(\rho, \delta)$ с параметрами $\rho<3 / 2$ и $\delta>1$. Такой выбор параметров не является исчерпывающим, прежде всего, это касается $\delta$. С небольшими изменениями техники результаты работы можно обобщить на случай более слабого условия $\delta>0$, для чего необходимо использовать более тонкие оценки интегралов типа $I_{2}$, оперируя с неабсолютно сходящимися интегралами. Условие на параметр $\rho$ тоже может быть ослаблено до $\rho<2$, что приведет к появлению дополнительных сингулярностей у функции Грина. Мы не приводим здесь анализа этих случаев во избежание чрезмерного увеличения объема статьи и планируем сделать это в будущих работах.

Благодарности. Работа поддержана СПбГУ (грант № 11.38.263.2014) и РФФИ (грант № 14-02-00326).

\section{Список литературы}

[1] Ю.Н. Демков, В.Н. Островский, Метод потенциалов нулевого радиуса в атомной физике, Изд-во ЛГУ, Л., 1975.

[2] Ф. А. Березин, Л. Д. Фаддеев, Докл. АН СССР, 137:5 (1961), 1011-1014.

[3] С. Альбеверио, Ф. Гестези, Р. Хёэг-Крон, Х. Хольден, Решаемъе модели в квантовой механике, Мир, М., 1991.

[4] Б. С. Павлов, ТМФ, 59:3 (1984), 345-353.

[5] Ю. А. Куперин, К. А. Макаров, Ю. Б. Мельников, ТМФ, 74:1 (1988), 103-111.

[6] J.F. Brasche, P. Exner, Yu. A. Kuperin, P. Šeba, J. Math. Anal. Appl., 184:1 (1994), $112-139$.

[7] J. Brüning, V. Geyler, K. Pankrashkin, J. Math. Phys., 46:11 (2005), 113508, 16 pp., arXiv: math-ph/0411078.

[8] S. Lakaev, M. Darus, Sh. Kurbanov, J. Phys. A: Math. Theor., 46:20 (2013), 205304, 15 pp.

[9] W. Bulla, F. Gesztesy, J. Math. Phys., 26:10 (1985), 2520-2528.

[10] J. Zorbas, J. Math. Phys., 21:4 (1980), 840-847.

[11] A. Grossmann, T. T. Wu, J. Math. Phys., 25:6 (1984), 1742-1745.

[12] I. A. Ivanov, J. Mitroy, J. Phys. B, 33:21 (2000), L831-L837.

[13] S. L. Yakovlev, C.-Y. Hu, D. Caballero, J. Phys. B, 40:10 (2007), 1675-1693.

[14] S. L. Yakovlev, V. A. Gradusov, J. Phys. A: Math. Theor, 46:3 (2013), 035307, 15 pp.

[15] А. Я. Повзнер, Матем. сб., 32(74):1 (1953), 109-156.

[16] Р. Ньютон, Теория рассеяния волн и частии, Мир, М., 1969.

[17] T. Ikebe, Arch. Rational Mech. Anal., 5:1 (1960), 1-34.

Поступила в редакцию 25.09.2014 